\title{
CAPM AWARDS
}

\section{Canadian Association of Professors of Medicine Awards}

The 2008 Core Medical Residents Research Awards were presented to Dr. Anna Mathew from the University of Western Ontario, Dr. Jeya Nadarajah of McMaster University, and Dr. Sara Stafford and Dr. Tara Sedlak from the University of British Columbia at the Annual Meeting of the Canadian Society for Clinical Investigation. The awards, co-sponsored by the CSCI and the Canadian Association of Professors of Medicine are to recognize outstanding research by core medicine residents and to highlight the importance of research participation as a component of the core medical training experience.
Clin Invest Med 2008; 31 (6): E410-E414.

\section{Elevated total bilirubin as a predictor of all-cause mortality in left-ventricular systolic dysfunction}

\section{Christopher Labos}

Department of Medicine, Montreal General Hospital, Faculty of Medicine,

McGill University, Montreal, Quebec

Introduction: Patients with heart failure (HF) often have elevated total bilirubin (TB) levels, which are often attributed to hepatic congestion. The prognostic value of this finding has not been evaluated.

We hypothesize that elevated TB levels are associated with increased rates of mortality, heart transplant and implantation of ventricular assist devices (VAD).

Methods: We retrospectively reviewed the charts of all patients followed at our heart failure center. We included all pts with left ventricular ejection fraction (LVEF) $<40 \%$. Elevated TB was defined as TB $>30 \mathrm{~mol} / \mathrm{L}$ (1.5 times the upper limit of normal). The primary endpoint was all-cause mortality. The secondary endpoint was a composite of death, transplant, and VAD implantation.

Results: Of 1020 patients with $\mathrm{LVEF}<40 \%, 162$ had elevated TB. The mean age (standard deviation) for patients with elevated TB vs. controls was 62 (14) vs. 67 (13) $(\mathrm{p}=0.04)$. The mean LVEF was similar, $23 \%(8)$ vs. $24 \%(8)$ for patiens with elevated TB vs controls. There were less females in the elevated TB than the control group (14.2\% vs. $25.2 \%, \mathrm{p}<0.001)$. There were 54 deaths, 5 transplants and 12 VADs in patients with elevated TB vs 183 deaths, 2 transplants and 6 VADs in controls. The median follow-up was 495 days (1st and 3rd quartiles: 110,1147) Elevated TB was independently associated with increased all-cause mortality, OR $1.88(95 \% \mathrm{CI} 1.32-2.80)$ and an increased composite endpoint of death/transplant/VAD, OR 1.96 (95\%CI 1.33-2.90). Age and LVEF were also independently associated with increased mortality with OR 1.05 (95\%CI 1.04-1.07) and OR 0.95 (95\%CI 0.94-0.97), respectively.

Conclusion: Risk of mortality, and the composite endpoint of death, transplantation, or VAD are doubled in patients with elevated TB compared to controls.

\begin{tabular}{l|c|c|c|c}
\hline & $\begin{array}{c}\text { Bilirubin } \\
\geq 30\end{array}$ & $\begin{array}{c}\text { Bilirubin } \\
<30\end{array}$ & $\begin{array}{c}\text { OR } \\
(95 \% \mathrm{CI})\end{array}$ & $\begin{array}{c}P \text { - } \\
\text { values }\end{array}$ \\
\hline Death (\%) & $54(33.3)$ & $183(21.3)$ & $1.88(1.32-2.80)$ & $<0.001$ \\
\hline $\begin{array}{l}\text { Death, VAD or } \\
\text { Transplant } \\
\text { combined (\%) }\end{array}$ & $57(35.2)$ & $188(21.9)$ & $1.96(1.33-2.90)$ & $<0.001$ \\
\hline
\end{tabular}


Identification of different clonal complexes and diverse amino acid substitutions in penicilin-binding protein 2 associated with borderline oxacillin resistance in Canadian staphylococcus aureus isolates

Jeya Nadarajah, M. Lee, D. Yamamura, M. McGavin

Dr. Jeya Nadarajah, Internal Medicine, McMaster University

Objective: Borderline oxacillin-resistant Staphylococcus aureus (BORSA) display reduced sensitivity to $\beta$-lactams, with oxacillin MIC values of $1-8 \mathrm{mcg} / \mathrm{mL}$. These isolates lack the mecA gene of methicillin-resistant Staphylococcus aureus. Their mechanisms of resistance vary and are yet to be fully elucidated. The aim of this study was to characterize clinical BORSA isolates and investigate their mechanisms of resistance.

Methods: A database of 38 Canadian BORSA isolates was assembled over six years. The BORSA isolates were characterized in terms of MIC, presence of $\beta$-lactamase, plasmids, phage type, pulsed-field gel electrophoresis (PFGE) profile and multilocus sequence type (MLST). Complete penicillin-binding protein $2(p b p 2)$ DNA sequences were determined. The $p b p 2$ gene from two strains (ma15 and msh12) were cloned and expressed in a susceptible strain. The PBP2 protein from ma15 and msh12 were purified by chromatography and assayed for binding capacity and kinetic rate constants.

Results: Among 38 BORSA, 26 had a common PFGE profile, and were MLST 25. The other isolates were genetically diverse. Amino acid substitutions occurred in the active transpeptidase domain of all BORSA. Plasmidmediated expression of the cloned BORSA $p b p 2$ genes in a sensitive strain, RN6390, raised its oxacillin MIC from 0.25 to $4 \mathrm{mcg} / \mathrm{mL}$, effectively rendering it borderline- resistant, while expression of $p b p 2$ from a susceptible strain had no effect. Kinetic studies showed that the PBP2 protein from BORSA isolates had lowered binding affinity to $\beta$ lactams.

Conclusions: Resistance was transferred to a susceptible strain of Staphylococcus aureus by expressing PBP2 from BORSA isolates. Different amino acid substitutions in $p b p 2$ contribute to borderline resistance. The predominant MLST 25 lineage was not related to any of the five clonal complexes of Staphylococcus aureus that contain MRSA, suggesting that these isolates cannot readily acquire $m e c A$ mediated resistance, and thus will likely remain treatable by $\beta$-lactam antibiotics.

\section{A step toward anti-MRSA phagotherapy}

\author{
Jeannot Dumaresq ${ }^{1}$, Sylvain Moineau ${ }^{2}$
}

Department of Medicine, Faculty of Medicine, Université Laval, ${ }^{1}$ and Department of Biochemistry and Microbiology, Faculty of Sciences and Engineering, Université Laval, ${ }^{2}$ Quebec, Canada

Objectives: The discovery of antimicrobial agents was one of the great triumphs of the 20th century. In less than 50 years, however, we have moved from the age of antibiotics to the age of antibiotic resistance. The phenomenon of antibiotic resistance has snowballed into a public health concern and alternatives to antibiotics are required. The aim of this project is to investigate phages as either therapeutic bioproducts or as prophylactic agents. As a preliminary step, we have identified phages infecting the main Canadian strains of methicillin resistant Staphylococcus aureus (CMRSA).

Materials and methods: Sixteen staphylococcal phages were obtained from the phage collection at the Université Laval (www.phage.ulaval.ca), and from international collaborators. Strains representing the ten most common CMRSA genotypes were purchased from the Institut National de Santé Publique du Québec. Among these ten bacterial strains was the main hospital acquired MRSA (CMRSA-2), and the main community associated strain (CMRSA-10). The 16 phages were tested on each CMRSA strain using the spot assay. The phage efficiency of plaquing was also compared between the CMRSA strains and their non-CMRSA host strains. Finally, the search for new staphylococcal phages was carried out by analyzing wastewater samples.

Results: Nine phages inactivated one or more strains of CMRSA. Globally, the ten CMRSA strains are sensitive to at least one phage. A new phage showing increased efficacy against CMRSA-10 was also generated. Finally, one phage isolate was recovered from the wastewater samples and was found to be effective against six of the ten CMRSA genotypes (including CMRSA-2 and CMRSA$10)$.

Conclusion: We have identified phages that possess a good anti-MRSA activity. The characterization of these phages is underway. It is our view that a better understanding of phage-bacteria interactions is absolutely required for bringing phage therapy into human clinical trials and acceptance. 


\section{Patients with chronic kidney disease and internal carotid artery stenosis benefit from carotid en- darterectomy}

\section{A. Mathew ${ }^{1}$, M. Eliasziw, PJ Devereaux, J Merino, H.J.M \\ Barnett, A.X Garg for the North American Symptomatic Carotid Endarterectomy Trial (NASCET) Collaborators.}

${ }^{1}$ Department of Medicine, The University of Western Ontario $\sim$ Schulich School of Medicine \& Dentistry

Current guidelines recommend carotid endarterectomy for patients with symptomatic high-grade (70-99\%) internal carotid artery stenosis, and for some patients with moderate-grade (50-69\%) stenosis. Proportionately fewer chronic kidney disease (CKD) patients undergo carotid endarterectomy compared to patients with normal renal function. To study the effect and safety of endarterectomy in CKD patients, we re-analysed the North American Symptomatic Carotid Endarterectomy Trial (NASCET), a global study involving 106 surgical centers. All patients received best medical care and one-half were randomized to endarterectomy. Glomerular filtration rate (eGFR) was estimated for $98 \%$ of participants: 524 patients with CKD defined by an eGFR less than $60 \mathrm{~mL} / \mathrm{min}$ per $1.73 \mathrm{~m}^{2}$ (mean 49, min 19) and 966 patients without CKD (eGFR of $60 \mathrm{~mL} / \mathrm{min}$ per $1.73 \mathrm{~m}^{2}$ or more). The primary outcomes were ipsilateral stroke and all cause mortality at 2 years.

For medically-treated patients with high-grade stenosis, the 2-year risk of ipsilateral stroke was higher in CKD patients than those without CKD $(31.6 \%$ vs $19.3 \%, p=0.01)$. In the group with high-grade stenosis, the absolute risk reduction (ARR) of ipsilateral stroke with carotid endarterectomy was greater for those with CKD compared to those without CKD (26\% [95\% CI 15.1 to 36.8], $\mathrm{p}<0.001$ vs $9.8 \%$ [95\% CI 1.9 to 17.7], $p=0.047$; test for interaction, $p=0.018$ ). The benefit was less apparent among patients with moderate-grade stenosis, with and without CKD. Perioperatively, CKD patients had similar rates of death or stroke compared to those without CKD, but had higher risks of myocardial infarction, congestive heart failure, or arrhythmia.

In conclusion, CKD patients with symptomatic high-grade carotid stenosis have at least a similar, and likely a larger benefit in stroke reduction after endarterectomy compared to those without CKD. Our results help inform decisionmaking by physicians and their patients about the appropriateness of endarterectomy.

\section{Validation of predictive models for germline muta- tions in DNS mismatch repair: Genes in colorectal cancer}

\section{Jose Monzon}

University of British Columbia, Department of Medicine

Background: Hereditary Non-Polyposis Colorectal Cancer (HNPCC) Syndrome is defined by the presence of germline mutations in mismatch repair genes. Identifying families that harbour these mutations is crucial for genetic counseling and cancer prevention. Models have been devised that predict mutation carrier status. The performance of these models on an independent cohort has yet to be evaluated.

Methods: amilies referred to the Hereditary Cancer Program (HCP) in British Columbia, which were at risk for harbouring an HNPCC mutation, underwent genotyping, immuno-histochemistry and/or microsatellite instability testing. The clinical data obtained from these families was used to calculate model-derived probabilities of harbouring an HNPCC germline mutation in the proband.

Results: Genetic testing was completed on 117 probands. Seventy-three probands had records available to retrieve the necessary data fields to input into the models. Twentyseven patients were mutation positive $(35.6 \%)$ and 46 were mutation negative $(64.4 \%)$. The mutation positive probands more frequently had a younger age at diagnosis, right-sided tumours, personal and family history of endometrial cancer, and a family history of CRC (all had a $\mathrm{p}<0.05$ ). The ROC curves for the various models had the following concordance indexes: $0.75(95 \% \mathrm{CI} ; 0.64-0.69), 0.84$ (0.74-0.94), 0.85 (0.76-0.94), and $0.86(0.76-0.96)$ for the Myriad, Edinburgh, Wijnen, and MMRpro models respectively. The Amsterdam II (AII) criteria had a sensitivity and specificity of 0.704 and 0.739 respectively. The performance characteristics of all models, except for the Myriad, outperformed the AII criteria at selecting individuals who may carry a mutation.

Conclusions: In probands that fulfill referral criteria for HNPCC screening at the HCP in BC, the Edinburgh, Wijnen and MMRpro models are equivalent to each other and better than the AII criteria at predicting the probability of a MMR germline mutation. Given the similar predictive performance, the choice of a preferred tool will depend upon ease of use and flexibility. 
Epidemiology of staphylococcus aureus nasal colonization and influence on outcome in the critically ill

\author{
Daniel J. Niven ${ }^{1}$, Kevin B. Laupland ${ }^{1-4}$, Daniel B. Greg- \\ son $^{1,2}$, Deirdre L. Church ${ }^{1,2}$ for the S. aureus screening ini- \\ tiative group
}

Departments of Medicine ${ }^{1}$, Pathology and Laboratory Medicine $^{2}$, Critical Care Medicine ${ }^{3}$, Centre for Antimicrobial Resistance ${ }^{4}$, University of Calgary, Calgary Health Region, and Calgary Laboratory Services, Calgary, Alberta, Canada.

Introduction: Nosocomial Staphylococcus aureus infections are associated with significant morbidity and mortality in critically ill patients. The objective of this study was to determine the rate of $S$. aureus nasal colonization at admission to intensive care units (ICU) and assess its effect on the development of an ICU-acquired $S$. aureus infection.

Methods: We screened all ICU admissions for nasal colonization within the Calgary Health Region from October 2005 to September 2006 and followed patients to hospital discharge or death or S. aureus infection to 30-days.

Results: There were 1308 patients admitted to ICU for $>48$ hours and screened for nasal colonization. Fifty (4\%) were methicillin-resistant (MRSA) positive, 311 (24\%) were methicillin-sensitive (MSSA) positive, and 947 (72\%) were nasal screen negative. Overall, 5\% (63/1239) of patients uninfected at ICU admission developed an ICU-acquired $S$. aureus infection. The rate of infection was 5\% in MRSA colonized patients, $12 \%$ in MSSA colonized patients, and $3 \%$ in non-colonized patients. A positive nasal screen [odds ratio (OR) 4.7, 95\% confidence interval (CI) 2.7-7.9], neuro/trauma patients (OR 3.1, 95\% CI 1.8-5.2), and higher first Therapeutic Intervention Scoring System score (OR 1.03 per point, 95\% CI 1.01-1.05) were independent predictors for developing an ICU-acquired $S$. aureus infection.

Conclusion: Nasal colonization with $S$. aureus is a significant risk factor for ICU-acquired $S$. aureus infections.
Progestational and androgenic activity of the oral contraceptive and angiotensin-dependent control of the renal circulation

Magdalena A. Sarna ${ }^{1}$, Norman K. Hollenberg ${ }^{2}$, Ellen W. Seely ${ }^{3}$, Sofia B. Ahmed ${ }^{1,4}$

Department of Medicine ${ }^{1}$, University of Calgary, Calgary, Canada; Departments of Medicine ${ }^{2}$ and Radiology ${ }^{3}$, Brigham and Women's Hospital, Harvard University, Boston, United States; Alberta Kidney Disease Network ${ }^{4}$.

Introduction: Oral contraceptive (OC) use is associated with increased intra-renal renin angiotensin system (RAS) activity and risk of nephropathy. However, individual contributions of progestin and estrogen in the OC have not been elucidated.

Methods: Eighteen OC users ( 8 healthy, 10 Type I diabetes mellitus (DM)) were studied in high salt balance, a state of maximal RAS suppression. Progestational and androgenic activities of the progestin in each OC were standardized to the activity of the reference synthetic progestin norethindrone. Renal plasma flow (RPF) was measured by paraaminohippurate clearance at baseline and in response to angiotensin converting enzyme (ACE)-inhibition with captopril. The change in RPF represented the level of angiotensin-dependent control of the renal circulation.

Results: As anticipated, all subjects demonstrated a renal vasodilatory response to ACE-inhibition $(p=0.0005)$, which did not differ by diabetic status $(\mathrm{p}=0.6)$. While no association was observed between the dose of ethinyl estradiol and the RPF response to captopril $(\mathrm{r}=-0.14, \mathrm{p}=0.6)$, there was a positive correlation between increasing $\mathrm{OC}$ progestational activity and the RPF response to ACE-inhibition ( $r=0.52$, $\mathrm{p}=0.03$ ). Similar results were noted with increasing OC androgenic activity $(\mathrm{r}=0.60, \mathrm{p}=0.008)$. A multiplicative effect of increased progestational and androgenic activity on the RPF response to ACE-inhibition was observed $(p=0.01)$. On sub-group analysis, only healthy subjects showed an association between progestational activity and angiotensin-dependent control of the renal circulation $(\mathrm{r}=0.71, \mathrm{p}=0.05$ healthy subjects; $\mathrm{r}=0.14, \mathrm{p}=0.7$ diabetics; $\mathrm{p}=0.07$ between groups). Similar results were noted with respect to androgenic activity $(\mathrm{r}=0.83, \mathrm{p}=0.01$ healthy subjects; $r=-0.27, p=0.4$ diabetics; $p=0.01$ between groups).

Conclusion: Angiotensin-dependent control of the renal circulation is positively correlated to the level of progestational and androgenic activity of the progestin component of the OC, though these findings may not apply to women with Type $1 \mathrm{DM}$. When choosing an OC, women at risk of kidney disease may consider formulations with low progestational and androgenic activity. 
Evaluation of resident attitudes and self-reported competencies in health advocacy

\section{Dr. Tara Sedlak, Dr. Sara Stafford}

University of British Columbia, Department of Medicine

Background: Health advocacy, a CanMEDS role mandated by the Royal College, is the responsibility of physicians to use their expertise/influence to advance the wellbeing of patients, communities, and populations. There is limited baseline data on resident attitudes or self-reported competencies on this subject.

Objective: To describe resident attitudes and self-reported competencies towards health advocacy.

Methods: We conducted a literature review on health advocacy education and found limited information. To construct an advocacy survey, we conducted a pilot experience with 7 residents participating in a community health promotion event. The residents provided narrative feedback and the information was used to generate items of the survey. Face validity was established by having the same resi- dents review the survey. Content validity was established by inviting an expert physician panel to review the survey. Concurrent validity could not be assessed due to lack of a gold standard. The refined survey was then distributed to a cohort of core Internal Medicine residents electronically.

Results: The survey was completed by 76 residents with a response rate of $68 \%$. The majority agreed to accept an advocacy role for societal health needs beyond caring for individual patients. Most confirmed their ability to identify health determinants and reaffirmed the inherent requirements for health advocacy. Multiple target populations were identified as potential beneficiaries. Slightly over half $(56 \%)$ of residents reported increased competencies on health advocacy, although $29 \%$ reported neutrality. However, $76 \%$ residents reported no current engagement in advocacy activity, and 36\% were undecided if they would engage in advocacy during their remaining time as residents, fellows or staff. The common barriers reported include time, rest and stress.

Conclusions: Athough Internal Medicine residents endorsed the importance to be health advocates and reported possessing the competencies, most are not actively involved during residency due to time, stress and limited rest. 